\title{
patients with relapse
}

Grégory GATOUILLAT ${ }^{1,2}$, Delphine GIUSTI ${ }^{1,2}$, Sébastien LE JAN ${ }^{1}$, Julie PLEE ${ }^{1,3}$, Philippe BERNARD ${ }^{1,3}$, Frank

ANTONICELLI ${ }^{1}$ and Bach Nga PHAM ${ }^{1,2}$

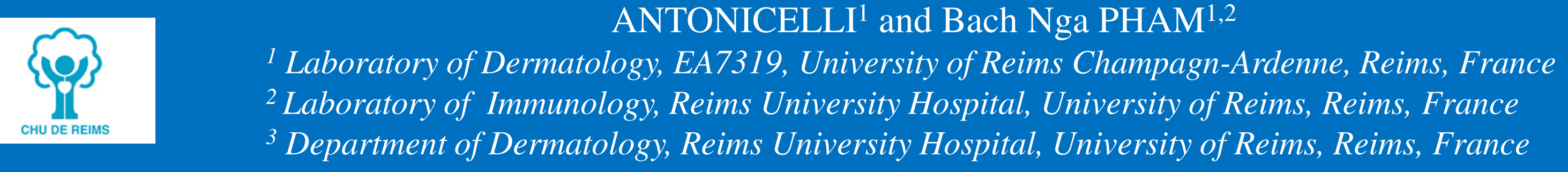

\section{INTRODUCTION}

\section{BACKGROUND}

Bullous Pemphigoid (BP) is an autoimmune bullous disease characterized by tense blisters, widespread erythema, and autoantibodies against the hemidesmosomal proteins BP180 and BP230. Most of BP patients are clinically controlled upon treatment with corticosteroids; however, approximately $30 \%$ of BP patients experience relapse during the first year of treatment. Noteworthy, BP patients may present several distinct presentations. Atypical manifestations include mucous membrane alterations, which mimic epidermolysis bullosa acquisita (EBA), another AIBD characterized by autoantibodies against type VII collagen (collVII).

\section{OBJECTIVE}

- To determine whether detection of anti-collVII antibodies could help in better characterizing BP patients with mucosal involvement.

- To investigate whether the clinical outcome of BP patients could be associated with the occurrence of anti-collVII autoantibodies

\section{PATIENTS AND METHODS}

Seventy-one consecutive patients with a new diagnosis of BP were included in this observational study conducted by the dermatology department of Reims University Hospital (French reference center for auto-immune bullous skin diseases). Patients included 47 BP without mucosal involvement and 24 BP with mucosal involvement. Clinical and serological data were recorded at the time of diagnosis (Table 1). Patients were followed, when possible, for at least one year. All patients gave their informed and written consent before participating to the study in accordance with the Helsinki declaration.

Evaluations of serological concentrations of anti-collVII, anti-BP180 and antiBP230 were performed by specific ELISA (MBL International, Japan).

Statistical analyses were performed for all quantitative measures by using nonparametric tests (Mann-Whitney, Fisher's exact test). Differences were considered significant when $P$ values were 0.05 or less.

\section{Table 1. Clinical and serological characteristics of BP patients}

\begin{tabular}{lcccc}
\hline & Total BP & BP without mucosal involvement & BP with mucosal involvement & $P$ value \\
\hline Patients No & 71 & 47 & 24 & $75.8 \pm 12.6(45-92)$ \\
Mean age \pm SD (range), years & $80.3 \pm 10.1(45-95)$ & $82.6 \pm 7.9(53-95)$ & 1.2 & $\mathbf{0 . 0 5}$ \\
Sex ratio (female/male) & 1.7 & 2.1 & $61.3 \pm 29.7$ \\
Total BPDAI (mean \pm SD) & $46.0 \pm 27.4$ & $38.0 \pm 22.9$ & $57.9 \pm 28.2$ & 0.25 \\
Skin BPDAI (mean \pm SD) & $44.8 \pm 26.1$ & $38.0 \pm 22.9$ & $11(45.8)$ & $\mathbf{0 . 0 0 1}$ \\
Relapse, patients No (\%) & $24(33.8)$ & $13(27.6)$ & 0.12
\end{tabular}

Serological data

Anti-colVII antibodies

Positive ELISA value, No (\%)

Mean $\pm \mathrm{SD}(\mathrm{U} / \mathrm{mL})$

Anti-BP180 antibodies

Positive ELISA value, No (\%)

Mean $\pm \mathrm{SD}(\mathrm{U} / \mathrm{mL})$

Anti-BP230 antibodies

Positive ELISA value, No (\%)

Mean $\pm \mathrm{SD}(\mathrm{U} / \mathrm{mL})$

\begin{abstract}
$2(2.8)$
$2.6 \pm 1.8$
\end{abstract}

$59(83.1)$

$67.1 \pm 50.0$

33 (46.5)

$26.2 \pm 36.9$
$0(0.0)$

$2.4 \pm 1.3$

$40(85.1)$

$65.8 \pm 48.6$

$26(55.3)$

$31.4(39.7)$
$2(8.3)$

$3.1 \pm 2.6$

$19(79.2)$

$69.5 \pm 56.2$

7 (29.2)

$16.1 \pm 28.8$
0.11

0.29

0.52

0.80

0.046
Figure 1. Variations in anti-collVII antibodies concentrations according to disease outcome
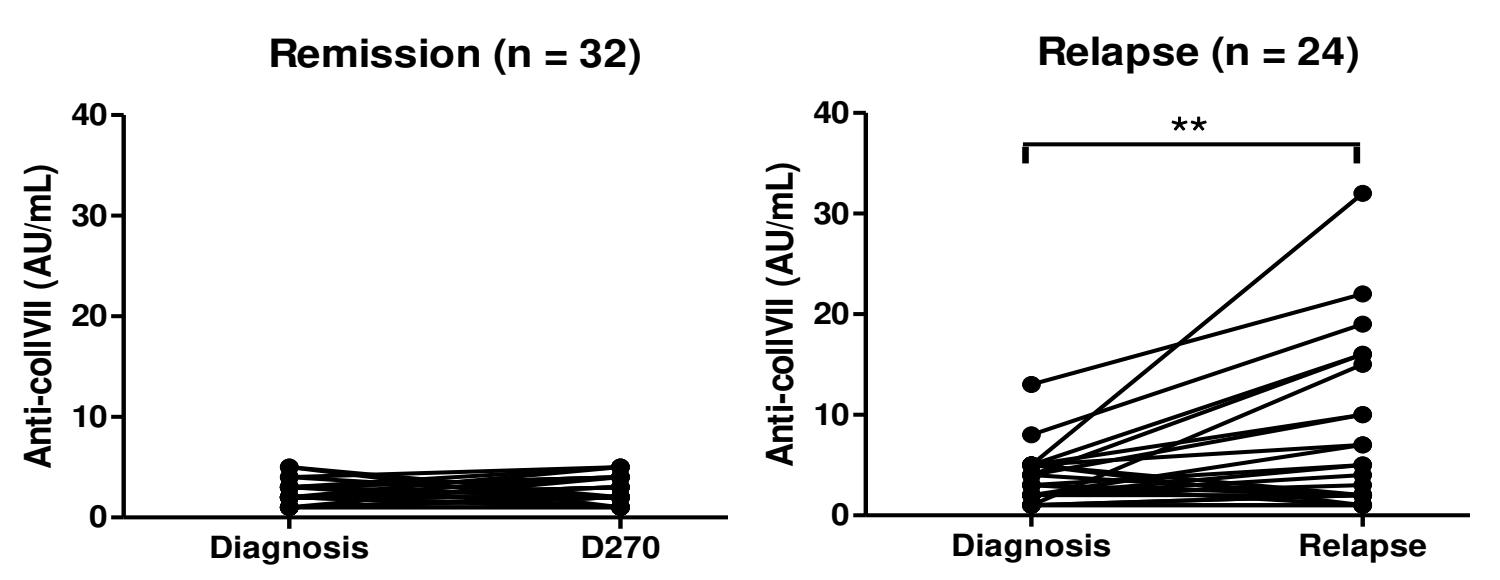

Serum levels of anti-collVII antibodies in patients with ongoing remission and patients with relapse. Median delay between diagnosis and relapse was 270 days. Comparison was made either between baseline and D270 for patients with ongoing remission or between baseline and relapse ${ }^{\star \star} \mathrm{P}<0.01$.

\section{CONCLUSION}

These results demonstrate that the detection of anti-collVII antibodies is not useful to distinguish BP with mucosal involvement from EBA at baseline (Table 1), but identifies a subgroup of BP patients at time of relapse (Fig 1 and 2).

The appearance of anti-collVII antibodies occurred in BP patients who also displayed a sustained serum level of anti-BP180 antibodies at time of relapse (Fig 2), but was independent of mucosal involvement at time of diagnosis.

Further investigations are required to demonstrate whether the occurrence of anti-collVII is related to an epitope spreading mechanism in this subpopulation of BP patients who relapsed, and to assess the clinical relevance of the presence of anti-CollVII antibodies in the serum of those BP patients.

\section{Figure 2. Serological findings in relapsing BP patients}

Patients with increased concentration of anti-collVII antibodies

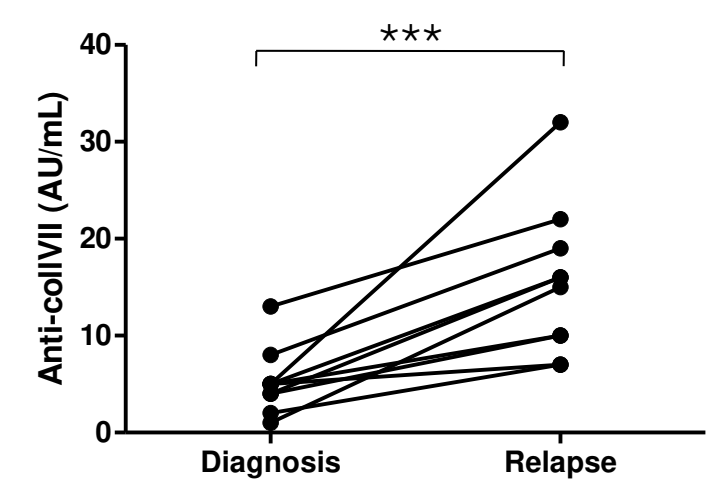

Patients without anti-collVII antibodies
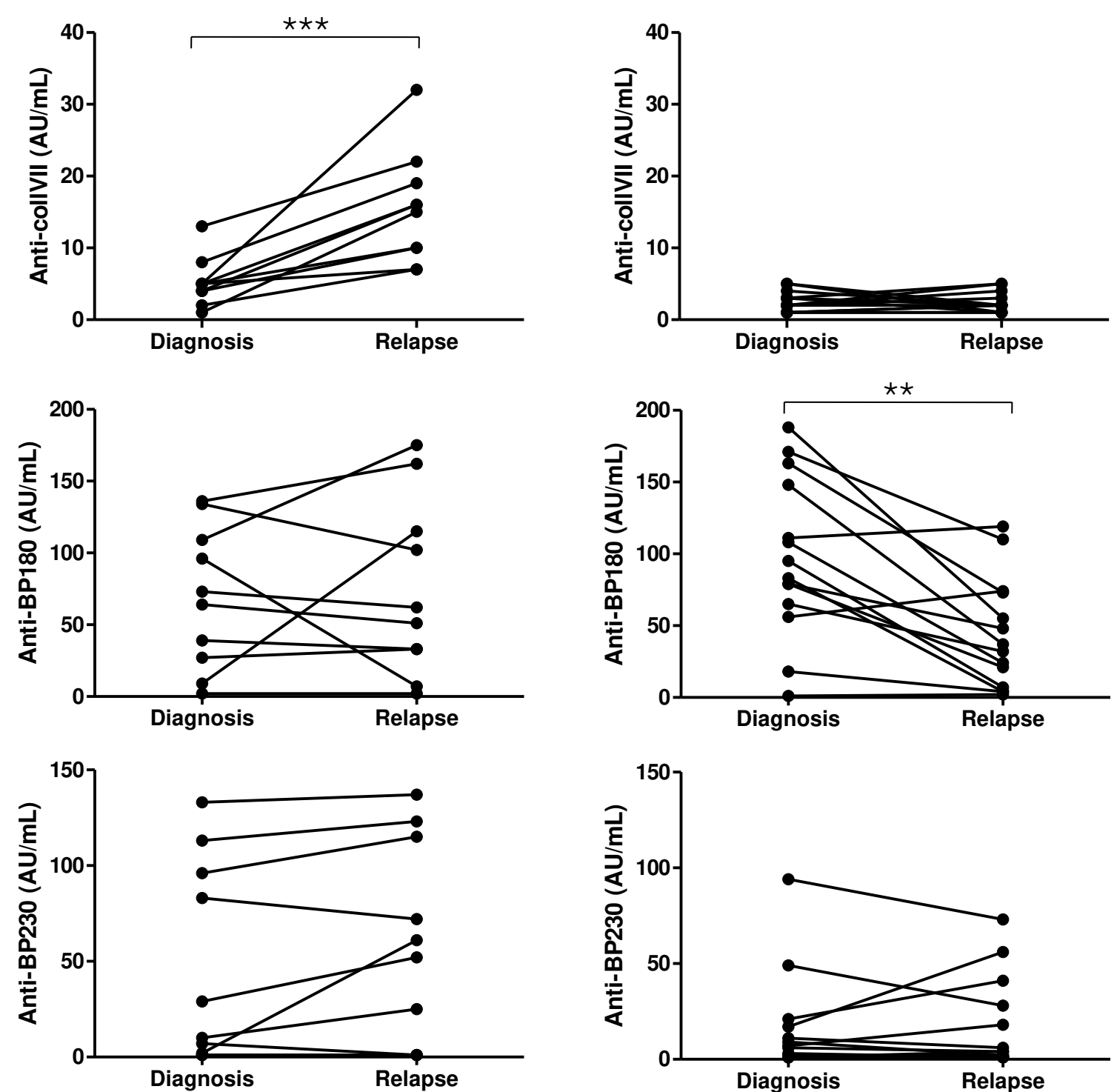

Serum levels of anti-BP180, anti-BP230 and anti-collVII antibodies in the 24 relapsing patients. Comparison was made between baseline and relapse either in patients with increased concentrations of anti-collVII or in patients without detectable anti-collVII antibodies. ${ }^{*} * \mathrm{P}<0.01,{ }^{* \star *} \mathrm{P}<0.001$ 